\title{
Focus on Gastrointestinal Tumors
}

Gastrointestinal tumors, from the commonly presenting neoplasms of the stomach and large intestine to the rarer lesions within the small intestine, are an area of keen professional interest. Indeed, research in this area generates a considerable body of scientific literature covering the entire spectrum from bench to bedside. Yet gastrointestinal tumors still pose a number of major conceptual and practical challenges. Lesions in the small intestine, for example, often elude early detection and their general symptoms frequently perplex clinicians. We are a long way from completely understanding the origin and differentiation of the various tumors of the digestive tract, and thus, prediction of their clinical behavior is still a major hurdle. The combination of surgery and chemotherapy or radiation therapy, although successful in some areas, is ineffective for treating other types of tumors.

The discovery of the genetic origins of hereditary colorectal cancer dates back to the 1980s and has since served as a paradigm for the study of the genetics, pathobiology and therapy of other types of cancers. Since the cloning of the FAP gene, research in the field of gastrointestinal tumors has made great strides. Our deeper knowledge of the genetics and pathobiology of these tumors has yielded breakthroughs in diagnostic and therapeutic regimens, extending patient survival and improving quality of life. However, there is still much work that needs to be done to improve the prognosis of patients diagnosed with these malignancies.

In this sea of information, what landmark should serve as our guide? Our future direction will be dictated by the technological advances converging across all disciplines: from the identification of novel receptors and kinases involved in tumorigenesis, to the application of targeted therapies, to breakthroughs in surgical techniques. Contemporary imaging and endoscopic methods, including computed tomography, magnetic resonance imaging and optical fiber technology, will facilitate the early detection of lesions. The transdisciplinary application of research and clinical data brings us ever closer to making personalized medicine a reality, improving the quality of life and clinical outlook for each patient.

We thank all the authors who contributed their articles to the first issue of this journal. In order to sustain the high quality of our publications and maintain the interest for a broad audience, we encourage submissions of high-quality manuscripts from the scientific and medical community.

With this, we extend a warm welcome to our readers of Gastrointestinal Tumors. We hope that our journal will provide a platform that unites stimulating and insightful articles across all disciplines, converging to advance the frontiers of research into the biology, diagnosis and treatment of these fascinating tumors.

Jing-Yuan Fang, Shanghai Peter Malfertheiner, Magdeburg 